\title{
OBTENÇÃO DE QUITOSANA A PARTIR DE CARAPAÇAS DE SIRI
}

\section{OBTAINMENT OF CHITOSAN FROM CRAB SHELLS}

Raquel Farias Weska ${ }^{1}$; Jaqueline de Motta Moura ${ }^{2}$; Jaques Rizzi ${ }^{3}$; Luiz Antonio de Almeida Pinto ${ }^{4}$

${ }^{1}$ Universidade Federal do Rio Grande - FURG - Rio Grande - Brasil raquelweska@terra.com.br

${ }^{2}$ Universidade Federal do Rio Grande - FURG - Rio Grande - Brasil

${ }^{3}$ Universidade Federal do Rio Grande - FURG - Rio Grande - Brasil

${ }^{4}$ Universidade Federal do Rio Grande - FURG - Rio Grande - Brasil

\section{Resumo}

A quitosana é um biopolímero derivado da quitina, que é encontrada na carapaça e exoesqueleto de crustáceos e outras fontes. $O$ objetivo deste trabalho foi a obtenção de quitosana a partir de carapaças de siri, sendo esta caracterizada pela massa molecular, que é um dos parâmetros que define suas aplicações específicas. A quitina foi obtida pelas etapas de desmineralização, desproteinização e desodorização, e a quitosana foi produzida por desacetilação alcalina. A estimativa da viscosidade intrínseca da quitosana foi feita através de uma regressão linear com os valores da viscosidade reduzida e da concentração, utilizando a equação de Huggins, com $R^{2} \geq$ 0,99. A massa molecular da quitosana foi calculada pela equação de Mark-Houwink-Sakurada, $e$ foi semelhante à da quitosana obtida por resíduos de camarão, em torno de $160 \mathrm{kDa}$.

Palavras-chave: biopolímero, resíduos de pescado, massa molecular.

\section{Introdução}

A quitina é um dos mais abundantes amino polissacarídeos naturais. As principais fontes comerciais da quitina são os resíduos de camarão, lagosta e siri.

O siri apresenta em sua composição 15 a $20 \%$ de quitina, que pode ser extraída e convertida em quitosana por meio de uma hidrólise alcalina, podendo ocasionar uma desacetilação incompleta, e também em uma despolimerização de extensões variáveis, resultando em quitosanas com diferentes massas moleculares. A aplicação deste polímero é limitada pela sua elevada massa molecular, resultando numa baixa solubilidade em meio aquoso.

O objetivo do trabalho foi a obtenção de quitosana a partir de carapaças de siri, sendo caracterizada a partir da determinação da massa molecular. 


\section{Materiais e métodos}

\subsection{Obtenção de quitina e produção de quitosana}

A matéria prima utilizada foi carapaças de siri (Maia squinado), obtidas das indústrias pesqueiras locais, e após uma pré-seleção foram moídas até o diâmetro médio de $4 \mathrm{~mm}$ em moinho de facas Wiley Mill - motor 1,1kW.

O processo utilizado para a obtenção de quitina a partir de carapaças de siri, foi semelhante ao utilizado por Soares et al. (2003), para resíduos de camarão, com modificações na etapa de desmineralização, segundo Moura et al. (2004). A quitina obtida foi seca em um secador de bandejas durante 4 horas, a $80^{\circ} \mathrm{C}$.

A desacetilação da quitina para a produção de quitosana foi realizada em escala de bancada, utilizando solução alcalina concentrada $\left(45^{\circ} \mathrm{Bé}\right)$, na temperatura de $130^{\circ} \mathrm{C}$, com $1,5 \mathrm{~h}$ de duração, sob agitação constante.

A purificação da quitosana foi realizada segundo procedimento semelhante ao descrito por Roberts et al. (1992). A quitosana foi dissolvida em solução de ácido acético, centrifugada para a retirada do material não dissolvido, precipitada com adição de solução de $\mathrm{NaOH}$ até $\mathrm{pH}$ 12,5, neutralizada com ácido acético até pH 7 e centrifugada para a retirada do sobrenadante.

As análises de composição centesimal (umidade, cinzas, N-proteína, N-quitina e Nquitosana), foram realizadas segundo os métodos da AOAC (1995).

\subsection{Determinação da massa molecular da quitosana}

As viscosidades das soluções de quitosana em ácido acético $0,1 \mathrm{M} /$ cloreto de sódio $0,2 \mathrm{M}$, em cinco concentrações $\left(0,1\right.$ a 1,2 g. $\left.\mathrm{L}^{-1}\right)$, a $25^{\circ} \mathrm{C}$, foram medidas em um viscosímetro capilar CannonFenske. A viscosidade intrínseca [ $\eta$ ] foi estimada por ajuste da equação de Huggins (Equação 1) aos resultados obtidos.

$$
\eta_{\mathrm{sp}} / \mathrm{c}=[\eta]+\mathrm{k} \cdot[\eta]^{2} \cdot \mathrm{c}
$$

onde que $\eta_{\mathrm{sp}} / \mathrm{c}$ é a viscosidade reduzida, $\eta_{\mathrm{sp}}$ é a relação entre a viscosidade do polímero em solução e o solvente, c é a concentração da solução e k é uma constante válida para cada polímero, conforme Alsarra et al. (2002).

A massa molecular viscosimétrica média $\left(\mathrm{M}_{\mathrm{V}}\right)$ da quitosana foi calculada a partir do valor da viscosidade intrínseca usando a equação de Mark-Houwink-Sakurada (Equação 2), citada por Roberts \& Domszy (1982). 


$$
[\eta]=K \cdot M_{v}{ }^{\alpha}
$$

em que K e $\alpha$ são constantes que dependem do sistema solvente-polímero.

\section{Resultados e discussão}

A Tabela 1 apresenta as análises de composição centesimal da quitina e da quitosana obtidas a partir de carapaças de siri.

Tabela 1- Resultados da composição centesimal da quitina e da quitosana.

\begin{tabular}{cccccc} 
& Umidade (b.u.\%) & Teor de cinzas (\%) & \multicolumn{2}{c}{ N-corrigido* (\%) } \\
& & & proteína & quitina & quitosana \\
\hline Matéria prima & $40,4 \pm 0,6$ & $40,8 \pm 0,3$ & $2,2 \pm 0,2$ & $17,2 \pm 0,8$ & - \\
Quitina & $89,8 \pm 0,6$ & $0,1 \pm 0,0$ & $0,3 \pm 0,0$ & $10,1 \pm 0,9$ & - \\
Quitosana & $95,4 \pm 0,5$ & -traços- & - & - & $4,5 \pm 0,5$ \\
\hline
\end{tabular}

* Fatores de conversão utilizados para proteína: 6,25; quitina: 14,5; quitosana: 11,5.

A partir da Tabela 1, observa-se que a desmineralização reduziu significativamente o teor de cinzas, e que o processo de obtenção da quitina a partir das carapaças foi eficiente, pois o rendimento de quitina em relação à matéria prima inicial de $16,6 \%$, estando dentro da faixa citada na literatura (Naczk et al., 2004).

O rendimento da quitosana obtida a partir das carapaças foi de $11,9 \%$, cerca de quatro vezes superior ao obtido de resíduos de camarão (Soares, 2003).

Figura 1 - Ajuste da equação de Huggins para os valores da viscosidade reduzida e concentração

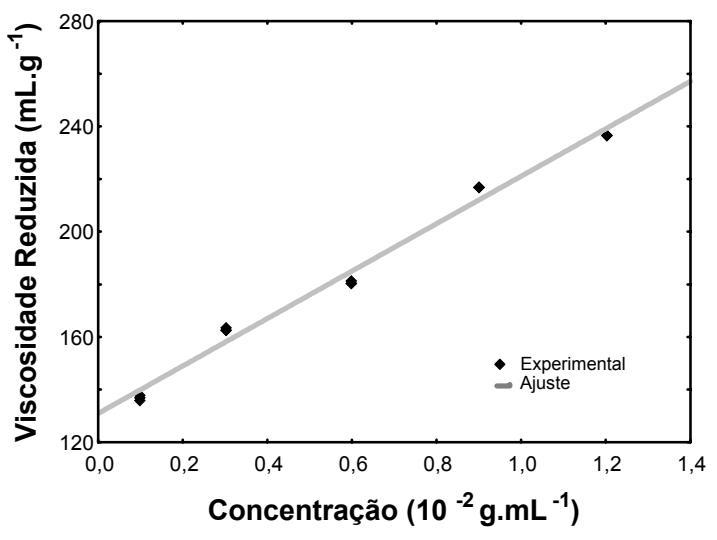

A Figura 1 apresenta o ajuste da Equação 1 para os valores da viscosidade reduzida e da concentração de quitosana, por regressão linear, utilizando o método dos mínimos quadrados. O 
bom ajuste aos dados experimentais foi verificado pelos altos coeficientes de correlação obtidos $\left(\mathrm{R}^{2}\right.$ $\geq 0,99)$.

A massa molecular viscosimétrica média da quitosana $\left(\mathrm{M}_{\mathrm{V}}\right)$ foi calculada pela Equação 2, com $[\eta]=129,94 \mathrm{~mL} \cdot \mathrm{g}^{-1}$, e com os valores de $\mathrm{K}=1,81 \cdot 10^{-3} \mathrm{~mL} \cdot \mathrm{g}^{-1}$ e $\alpha=0,93$, citados por Roberts \& Domszy (1982), obtendo-se um valor de $160 \mathrm{kDa}$, similar ao obtido por Soares para resíduos de camarão.

\title{
4. Conclusão
}

O teor de quitina obtida a partir de carapaças de siri ficou dentro da faixa citada pela literatura, com um rendimento de $16,6 \%$.

A quitosana de carapaças de siri apresentou um rendimento cerca de quatro vezes maior que a produzida com resíduos de camarão (Soares, 2003), sendo que a massa molecular ficou na mesma faixa, em torno de $160 \mathrm{kDa}$.

\begin{abstract}
Chitosan is a biopolymer derived from chitin, which is found crustacean's shells and exoskeletons, among other sources. The aim of this study was the obtainment of chitosan from crab shells, characterized by molecular weight, parameter which defines its specific applications. Chitin was obtained through the stages of demineralization, deproteinization and deodorization, and chitosan was produced by alkaline deacetilation. Chitosan's intrinsic viscosity was estimated by linear regression with the values of reduced viscosity and concentration, using Huggins equation, with $\mathrm{R}^{2}$ $\geq 0,99$. Chitosan's molecular weight was calculated by Mark-Houwink-Sakurada's equation, and it was similar to the chitosan obtained through shrimp's waste, around $160 \mathrm{kDa}$.
\end{abstract}

Key-words: biopolymer, fish wastes, molecular weight.

\section{Referências}

Assoc. of Official Analytical Chemists (AOAC) (1995). Official methods of analysis.

ALSARRA, A. et al. Molecular weight and degree of deacetylation effects on lipase-loaded chitosan bead characteristics. Biomaterials, v.23, p.3637-44, 2002.

ROBERTS, A.F. \& DOMSZY, J. G. Determination of the viscometric constants for chitosan. International Journal of Biological Macromolecules, v.4, p.374-77, 1982.

SOARES, N.M et al. Obtenção e purificação de quitosana a partir de resíduos de camarão em escala piloto. Revista Univap, São José dos Campos-SP, v.10, n¹8, p.88-92, junho 2003.

MOURA, J. M et al. Obtenção de quitosana a partir de resíduos de siri: otimização da etapa de desmineralização. In: II EQAMA. Anais do II EQAMA. Rio Grande: FURG, 2004. CD-ROM. 


\section{Primeiro autor:}

Nome completo: Raquel Farias Weska

Filiação institucional: Universidade Federal do Rio Grande

Departamento: Química

Endereço completo para correspondência (bairro, cidade, estado, país e CEP):

Rua Eng. Alfredo Huch, 475 - Cep: 96201-900 - Rio Grande/RS

e-mail: raquelweska@terra.com.br 